Int. J. Odontostomat., 7(2):179-184, 2013.

\title{
Malformaciones Craneofaciales en un Hospital Regional de Alta Complejidad
}

\author{
Craniofacial Malformation in a High Complexity Regional Hospital
}

\author{
Diego Mittersteiner ${ }^{*} \&$ Sergio Olate ${ }^{\star *, * \star *}$
}

MITTERSTEINER, D. \& OLATE, S. Malformaciones craneofaciales en un hospital regional de alta complejidad. Int. J. Odontostomat., 7(2):179-184, 2013.

RESUMEN: Las malformaciones craneofaciales son patologías complejas que afectan diferentes aspectos de la vida. El objetivo de esta investigación es identificar la prevalencia de malformaciones y las condicionantes en que se desarrollan en el Hospital Hernán Henríquez Aravena (HHHA) de Temuco. Se realizo una investigación en la Unidad de Neonatología del HHHA estudiando todos los ingresos con algún diagnostico de malformación craneofacial realizados entre enero de 2006 hasta agosto de 2010. Se estudiaron variables del paciente, de la madre y las características del diagnostico y el manejo del paciente. Los datos fueron analizados de forma descriptiva. Se observaron 44 casos con malformaciones craneofaciales, siendo un $56,82 \%$ de sexo masculino con un $22,73 \%$ de pertenencia a la etnia mapuche. La mayoría de los casos presentaron pesos adecuados para sus edades gestacionales, más del $30 \%$ de los casos fueron de pretérmino. El 52,27\% de las madres presentaron edades de parto menores de 30 años. El $50 \%$ de los casos se manejó en unidad de paciente crítico, $16 \%$ de los casos requirió cirugía durante su estadía en el servicio de neonatología y cerca del $70 \%$ de los casos egresaron vivos a su domicilio u otro hospital o unidad hospitalaria. Se concluye que el diagnostico de fisura labiopalatina es el mas prevalente y se determina la necesidad de manejo multidisciplinario para tratar casos complejos. La existencia de un promedio de 11 casos anuales exige un equipo activo en el área.

PALABRAS CLAVE: malformación, fisura palatina, sindrome craneofacial.

\section{INTRODUCCIÓN}

Las malformaciones craneofaciales son patologías complejas que, presentadas generalmente como síndromes, generan trastornos morfológicos, funcionales y psíquicos, con los consecuentes problemas del enfermo y su familia. Si bien, algunas de ellas pueden tener graves secuelas, un tratamiento correcto mejora la calidad de vida de quienes las padecen (Felemovicius \& Ortiz-Monasterio 2004; Múfalo et al., 2009; Roberts \& Shute, 2010; Gonzáles et al., 2003).

La literatura en Chile, presenta limitada información respecto de la epidemiología de las malformaciones craneofaciales, y los datos existentes corresponden a hospitales de la región Metropolitana y en su mayoría hacen referencia a diagnósticos de fisuras labiales, palatinas y labiopalatinas no sindromáticas (Nazer et al., 1995, 2001, 2003, 2010,
2003; Sepúlveda et al., 2008). Esta situación determina el desconocimiento de las condicionantes y relativiza la real importancia de estas patologías.

La región de la Araucanía, según el Censo 2002, representa el $29,6 \%$ de la población indígena total del país (INE-MIDEPLAN, 2005). El hospital Hernán Henríquez Aravena, es el centro de mayor complejidad de la región, y la unidad de neonatología reciben y maneja la mayoría de los recién nacidos con malformaciones craneofaciales provenientes de diversos sectores de la región (Olavarría, 2005).

El objetivo de esta investigación es identificar la prevalencia de malformaciones craneofaciales en la unidad de neonatología en el Hospital Hernán Henríquez Aravena en la IX región de la Araucanía.

\footnotetext{
Facultad de Medicina, Universidad de La Frontera, Temuco, Chile.

* CIMA, Facultad de Odontología, Universidad de La Frontera, Temuco, Chile.

${ }^{* * *}$ Centro de Investigación en Ciencias Biomédicas, Universidad Autónoma de Chile, Temuco, Chile.
} 


\section{MATERIAL Y MÉTODO}

Se realizó un estudio descriptivo retrospectivo, analizando la base de datos de egresos de la unidad de neonatología del Hospital Hernán Henríquez.

Se incluyeron todos los casos nacidos entre enero del año 2006 y agosto del año 2010, que presentaron alguno de los siguientes diagnósticos: fisura facial (facial, labial, palatina y facial labiopalatina), Disostosis craneofacial (síndrome de Goldenhart, síndrome de Treacher-Collins, síndrome de Pierre-Robin, Disostosis Cleidocraneal, síndrome de Nager y síndrome de Binder), Craneosinostosis simples (escafocefalia, Plagiocefalia, Braquicefalia, Trigonocefalia, Oxicefalia y Mixtas). Craneosinostosis complejas (síndrome de Crouzon, síndrome de Saethre-Chotzen, síndrome de Pfeiffer y síndrome de Apert). Aquellos casos que presentaron fisuras faciales en el contexto de alguna disostosis craneofacial o craneosinostosis, fueron contabilizados como disostosis craneofacial o craneosinostosis según corresponda.

Posteriormente se realizó una revisión en las fichas clínicas de cada caso. La información obtenida fue registrada en un formulario que consideraba variables como sexo y ascendencia étnica, adecuación de peso para edad gestacional y edad gestacional al nacimiento. También se incluyo variables maternas como la edad materna al momento del parto y variables de manejo incluyendo necesidad de manejo en unidad de paciente crítico independiente de la causa, cirugía durante estadía en servicio de neonatología independiente de la causa y condición de egreso del caso independiente de la causa.

Los datos obtenidos fueron agrupados y tabulados en una platilla de Excel para finalmente realizar el análisis de datos.

\section{RESULTADOS}

Tabla I. Distribución de casos por sexo, etnia y edad materna.

\begin{tabular}{lllllllll}
\hline \multirow{2}{*}{ Casos } & Sexo* & \multicolumn{5}{c}{ Etnia } & \multicolumn{5}{c}{ Edad materna } \\
\cline { 2 - 9 } & Masculino & Femenino & Presente & Ausente & Menos de 15 & $15-30$ & $31-40$ & Más de 40 \\
\hline $\mathrm{n}$ & 25 & 18 & 10 & 34 & 0 & 23 & 15 & 4 \\
\hline$\%$ & 56,82 & 40,91 & 22,73 & 77,27 & 0 & 52,27 & 34,09 & 9,09 \\
\hline
\end{tabular}

Tabla II. Distribución de malformaciones craneofaciales.

maciones craneofaciales entre enero del año 2006 y agosto del año 2010. Veinte y cinco sujetos fueron de sexo masculino $(56,82 \%), 18$ de sexo femenino $(40,91 \%)$ y en un caso no existía el dato $(2,27 \%)$. Diez casos pertenecieron a la etnia mapuche $(22,73 \%)$ y 34 casos no pertenecieron a ninguna etnia en particu$\operatorname{lar}(77,27 \%)$ (Tabla I).

Consideraciones del paciente y la madre. En 29 casos se observo un tamaño adecuado para su edad gestacional $(65,91 \%), 12$ casos presentaron un tamaño pequeño para su edad gestacional $(27,27 \%)$ y 3 de los casos presentaron un tamaño grande para su

\begin{tabular}{clll}
\hline Patología & \multicolumn{2}{l}{ Casos } \\
\cline { 3 - 4 } & & $\mathrm{n}$ & $\%$ \\
\hline Fisuras Faciales & 31 & 70 \\
No labiopalatinas & 0 & 0 \\
Labiopalatinas & 31 & 70 \\
Labial & 1 & 2 \\
Palatina & 7 & 16 \\
Labiopalatina & 23 & 52 \\
Encefaloce & 1 & 2 \\
Disostosis Craneofacial & 7 & 16 \\
Goldenhart & 4 & 9 \\
Treacher-Collins & 0 & 0 \\
Pierre robin & 2 & 5 \\
Disostosis & 1 & 2 \\
Cleidocraneal & & \\
Nager & 0 & 0 \\
Binder & 0 & 0 \\
Craneosinostosis & 5 & 11 \\
Simple & 1 & 2 \\
Compleja & 4 & 9 \\
Crouzon & 1 & 2 \\
Saethre-Chotzen & 0 & 0 \\
Pfeiffer & 1 & 2 \\
Apert & 2 & 5 \\
\hline
\end{tabular}


Tabla III. Distribución de Fisuras faciales, encefalocele y sindromes craneofaciales por sexo, etnia y edad materna.

\begin{tabular}{|c|c|c|c|c|c|c|c|c|c|c|c|c|c|c|}
\hline \multirow[t]{3}{*}{ Patología } & \multicolumn{4}{|c|}{ Sexo } & \multicolumn{4}{|c|}{ Etnia } & \multicolumn{6}{|c|}{ Edad materna } \\
\hline & \multicolumn{2}{|c|}{ Masculino } & \multicolumn{2}{|c|}{ Femenino } & \multicolumn{2}{|c|}{ Mapuche } & \multicolumn{2}{|c|}{$\begin{array}{l}\text { No } \\
\text { mapuche }\end{array}$} & \multicolumn{2}{|c|}{$15-30$} & \multicolumn{2}{|c|}{$31-40$} & \multicolumn{2}{|c|}{$\begin{array}{l}\text { Mayor de } \\
40\end{array}$} \\
\hline & $\mathrm{n}$ & $\%$ & $\mathrm{n}$ & $\%$ & $\mathrm{n}$ & $\%$ & $\mathrm{n}$ & $\%$ & $\mathrm{n}$ & $\%$ & $\mathrm{n}$ & $\%$ & $\mathrm{n}$ & $\%$ \\
\hline \multicolumn{15}{|l|}{ Fisura facial } \\
\hline \multicolumn{15}{|l|}{ Labial } \\
\hline Unilateral & 0 & 0 & 1 & 100 & 0 & 0 & 1 & 100 & 1 & 100 & 0 & 0 & 0 & 0 \\
\hline Bilateral & 0 & 0 & 0 & 0 & 0 & 0 & 0 & 0 & 0 & 0 & 0 & 0 & 0 & 0 \\
\hline Total & 0 & 0 & 1 & 100 & 0 & 0 & 1 & 100 & 1 & 100 & 0 & 0 & 0 & 0 \\
\hline \multicolumn{15}{|l|}{ Palatina* } \\
\hline Unilateral & 2 & 29 & 4 & 58 & 3 & 43 & 4 & 58 & 6 & 86 & 1 & 14 & 0 & 0 \\
\hline Bilateral & 0 & 0 & 0 & 0 & 0 & 0 & 0 & 0 & 0 & 0 & 0 & 0 & 0 & 0 \\
\hline Total & 2 & 29 & 4 & 58 & 3 & 43 & 4 & 58 & 6 & 86 & 1 & 14 & 0 & 0 \\
\hline \multicolumn{15}{|l|}{ Labiopalatina } \\
\hline Unilateral & 11 & 61 & 7 & 39 & 4 & 22 & 14 & 78 & 8 & 44 & 8 & 44 & 2 & 11 \\
\hline Bilateral & 3 & 60 & 2 & 40 & 1 & 20 & 4 & 80 & 3 & 60 & 1 & 20 & 1 & 20 \\
\hline Total & 14 & 61 & 9 & 39 & 5 & 22 & 18 & 78 & 11 & 48 & 9 & 39 & 3 & 13 \\
\hline Encefaloce ${ }^{* *}$ & 1 & 100 & 0 & 0 & 0 & 0 & 1 & 100 & - & - & - & - & - & - \\
\hline Total & 17 & 53 & 14 & 43 & 8 & 25 & 24 & 75 & 18 & 56 & 10 & 31 & 3 & 9 \\
\hline \multicolumn{15}{|l|}{ Síndrome Craneofacial } \\
\hline Goldenhar & 3 & 75 & 1 & 25 & 1 & 25 & 3 & 75 & 3 & 75 & 1 & 25 & 0 & 0 \\
\hline Pierre Robin & 2 & 100 & 0 & 0 & 0 & 0 & 2 & 100 & 0 & 0 & 1 & 50 & 1 & 50 \\
\hline D. Cleidocraneal & 1 & 100 & 0 & 0 & 0 & 0 & 1 & 100 & 1 & 100 & 0 & 0 & 0 & 0 \\
\hline Pfeiffer & 0 & 0 & 1 & 100 & 0 & 0 & 1 & 100 & 0 & 0 & 1 & 100 & 0 & 0 \\
\hline Crouzon & 1 & 100 & 0 & 0 & 1 & 100 & 0 & 0 & 0 & 0 & 1 & 100 & 0 & 0 \\
\hline Apert $^{* *}$ & 1 & 50 & 1 & 50 & 0 & 0 & 2 & 100 & 1 & 100 & 0 & 0 & 0 & 0 \\
\hline $\begin{array}{l}\text { Craneosinostosis } \\
\text { simple }\end{array}$ & 0 & 0 & 1 & 100 & 0 & 0 & 1 & 100 & 0 & 0 & 1 & 100 & 0 & 0 \\
\hline
\end{tabular}

*Variable sexo ausente en un caso ${ }^{* *}$ Variable edad materna ausente

edad gestacional $(6,82 \%)$. Quince de los casos nacieron de edades gestacionales menores a 37 semanas $(34,09 \%)$ y 29 con edades gestacionales entre 37 y 42 semanas $(65,91 \%)$. No se encontraron casos con edades gestacionales al nacimiento mayores de 42 semanas.

Respecto a las variables maternas (Tabla I), se encontraron 23 casos de edades maternas entre 15 y 30 años al momento del parto (52,27\%), 15 casos de edades maternas entre 31 y 40 años al momento del parto $(34,09 \%), 4$ casos con edades maternas mayores de 40 años al momento del parto $(9,09 \%)$ y en 2 casos no existía el dato $(4,55 \%)$.

Consideraciones en el manejo del paciente. Con respecto a las variables de manejo de los casos, en 22 casos fue necesario manejo en unidad de paciente crítico $(50 \%)$, mientras que en los restantes no fue necesario. El manejo quirúrgico no se indico en 37 casos $(84,09 \%)$, mientras que en 7 casos sí fue necesario $(15,91 \%)$. Respecto de los egresos, 19 casos egresaron vivos desde el servicio de neonatología hacia su domicilio (43,18\%), 12 casos egresaron vivos y fueron derivados a otro hospital o unidad $(27,27 \%)$ y 13 casos egresaron muertos $(29,55 \%)$.

Consideraciones del diagnóstico. Con respecto a las patologías detectadas (Tabla II), 31 casos presentaron fisuras faciales labio-palatinas $(70,45 \%)$ entre los cuales 1 caso presentó fisura labial (2,27\%), 7 casos presentaron fisuras palatinas $(15,91 \%), 23$ ca- 
Tabla IV. Distribución de fisuras y encefalocele de acuerdo a necesidad de manejo en unidad de paciente crítico (UPC), necesidad de manejo quirúrgico y condición de egreso

\begin{tabular}{|c|c|c|c|c|c|c|c|c|c|c|c|c|c|c|}
\hline \multirow[t]{3}{*}{ Patología } & \multicolumn{4}{|c|}{ Manejo en UPC } & \multicolumn{4}{|c|}{ Manejo quirúrgico } & \multicolumn{6}{|c|}{ Condición de egreso } \\
\hline & \multicolumn{2}{|c|}{$\begin{array}{l}\text { Requirió } \\
\text { UPC }\end{array}$} & \multicolumn{2}{|c|}{$\begin{array}{l}\text { No } \\
\text { Requirió } \\
\text { UPC }\end{array}$} & \multicolumn{2}{|c|}{$\begin{array}{l}\text { Requirió } \\
\text { Cirugía }\end{array}$} & \multicolumn{2}{|c|}{$\begin{array}{l}\text { No Requirió } \\
\text { Cirugía }\end{array}$} & \multicolumn{2}{|c|}{$\begin{array}{l}\text { Vivo a } \\
\text { domicilio }\end{array}$} & \multicolumn{2}{|c|}{$\begin{array}{l}\text { Vivo a otro } \\
\text { hospital o } \\
\text { servicio }\end{array}$} & \multicolumn{2}{|c|}{ Muerto } \\
\hline & $\mathrm{n}$ & $\%$ & $\mathrm{n}$ & $\%$ & $\mathrm{n}$ & $\%$ & $\mathrm{n}$ & $\%$ & $\mathrm{n}$ & $\%$ & $\mathrm{n}$ & $\%$ & $\mathrm{n}$ & $\%$ \\
\hline \multicolumn{15}{|l|}{ Fisura } \\
\hline \multicolumn{15}{|l|}{ Labial } \\
\hline Unilateral & 0 & 0 & 1 & 100 & 0 & 0 & 1 & 100 & 1 & 100 & 0 & 0 & 0 & 0 \\
\hline Bilateral & 0 & 0 & 0 & 0 & 0 & 0 & 0 & 0 & 0 & 0 & 0 & 0 & 0 & 0 \\
\hline Total & 0 & 0 & 1 & 100 & 0 & 0 & 1 & 100 & 1 & 100 & 0 & 0 & 0 & 0 \\
\hline \multicolumn{15}{|l|}{ Palatina } \\
\hline Unilateral & 5 & 71 & 2 & 29 & 1 & 14 & 6 & 86 & 2 & 29 & 3 & 42 & 2 & 29 \\
\hline Bilateral & 0 & 0 & 0 & 0 & 0 & 0 & 0 & 0 & 0 & 0 & 0 & 0 & 0 & 0 \\
\hline Total & 5 & 71 & 2 & 29 & 1 & 14 & 6 & 86 & 2 & 29 & 3 & 42 & 2 & 29 \\
\hline \multicolumn{15}{|l|}{ Labiopalatina } \\
\hline Unilateral & 7 & 39 & 11 & 61 & 3 & 17 & 15 & 83 & 9 & 50 & 4 & 22 & 5 & 28 \\
\hline Bilateral & 2 & 40 & 3 & 60 & 0 & 0 & 5 & 100 & 2 & 40 & 2 & 40 & 1 & 20 \\
\hline Total & 9 & 39 & 14 & 61 & 3 & 13 & 20 & 87 & 11 & 48 & 6 & 26 & 6 & 26 \\
\hline Encefaloce & 1 & 100 & 0 & 0 & 0 & 0 & 1 & 100 & 0 & 0 & 0 & 0 & 1 & 100 \\
\hline \multicolumn{15}{|l|}{$\begin{array}{l}\text { Síndrome } \\
\text { Craneofacial }\end{array}$} \\
\hline Goldenhar & 4 & 100 & 0 & 0 & 1 & 25 & 3 & 75 & 1 & 25 & 1 & 25 & 2 & 50 \\
\hline Pierre Robin & 2 & 100 & 0 & 0 & 1 & 50 & 1 & 50 & 1 & 50 & 1 & 50 & 0 & 0 \\
\hline D. Cleidocraneal & 0 & 0 & 1 & 100 & 0 & 0 & 1 & 100 & 1 & 100 & 0 & 0 & 0 & 0 \\
\hline Pfeiffer & 1 & 100 & 0 & 0 & 1 & 10 & 0 & 0 & 0 & 0 & 0 & 0 & 1 & 100 \\
\hline Crouzon & 1 & 100 & 0 & 0 & 0 & $\hat{0}$ & 1 & 100 & 0 & 0 & 1 & 100 & 0 & 0 \\
\hline Apert & 1 & 50 & 1 & 50 & 0 & 0 & 2 & 100 & 1 & 50 & 0 & 0 & 1 & 50 \\
\hline $\begin{array}{l}\text { Craneosinostosis } \\
\text { simple }\end{array}$ & 0 & 0 & 1 & 100 & 0 & 0 & 1 & 100 & 1 & 100 & 0 & 0 & 0 & 0 \\
\hline
\end{tabular}

sos presentaron fisuras labio-palatinas $(52,27 \%)$ (Tabla III).

En un caso se presentó encefalocele $(2,27 \%)$, 7 casos presentaron disostosis craneofacial $(15,91 \%)$ entre los cuales 4 casos presentaron síndrome de goldenhar $(9,09 \%), 2$ casos presentaron síndrome de Pierre-Robin $(4,55 \%)$ y 1 caso presentó disostosis cleidocraneal $(2,27 \%)$. En 5 casos se observo craneosinostosis $(11,36 \%)$ de los cuáles 1 caso presentó craneosinostosis simple $(2,27 \%), 4$ casos presentaron craneosinostosis complejas $(9,09 \%)$ entre las cuales un caso presentó síndrome de Crouzon $(2,27 \%)$, un caso presentó Síndrome de Pfeiffer $(2,27 \%)$ y 2 casos presentaron síndrome de Apert $(4,55 \%)$ (Tablas III y IV).

\section{DISCUSIÓN}

Las fisuras de labio y/o paladar son malformaciones congénitas de alta prevalencia en Chile. Se estima que por cada 740 nacimientos de niños vivos hay un afectado con dicha condición (Felemovicius \& Ortiz-Monasterio). Esta comprobación epidemiológica representa un problema de salud pública.

Las malformaciones craneofaciales en general son de las patologías malformativas más frecuentes del mundo, particularmente relevantes dentro de este grupo son las fisuras faciales (Sepúlveda et al.; Sze et al., 2002). En Chile las fisuras presentan una alta prevalencia (MINSAL, 2009). 
Nuestros resultados coinciden con Sze et al. y Navas et al. (2008), en el hecho de que las fisuras faciales son las alteraciones más prevalentes dentro de las malformaciones craneofaciales.

Para Sepúlveda et al., con respecto las fisuras labiales y palatinas, el grupo más frecuente es el de las fisuras labiopalatinas, le siguen las fisuras palatinas y en tercer lugar las fisuras labiales. Lo anterior coincide con los resultados obtenidos en este trabajo, sin embargo, las proporciones de cada grupo son diferentes a las nuestras. Las variaciones de las poblaciones estudiadas pueden ser la causa a estas diferencias.

Ya Navas et al., y Sze et al., señalaron que las disostosis craneofaciales presentan una mayor frecuencia que las craneosinostosis lo que concuerda con nuestro trabajo. La patología de estos grupos, que presentó la mayor frecuencia fue el síndrome de Goldenhart, siendo casi un 10\% de del total de los casos. Le siguen en frecuencia el síndromede Pierre Robin y de Apert, siendo cada uno alrededor de un $5 \%$ del total. Los problemas en cuanto a diferenciar patologías asociadas a craneosinostosis o a disostosis craneofaciales, permite que algunos autores determinen una patología en un grupo o en otro, lo cual dificulta las comparaciones con otras investigaciones.

Del total de malformaciones craneofaciales, un $56,82 \%$ de los casos era de sexo masculino y el sexo femenino se encontraba en un $40,91 \%$ de los casos y en un caso no existía el dato (2,27\%). Esta inclinación hacia el sexo masculino es coincidente con lo plateado por Alderman et al., (1988), aunque puede responder también a variaciones de los grupos de muestra.

Para Alderman et al., la edad materna se describe como factor de riesgo para el desarrollo de malformaciones craneofaciales, sin embargo en nuestra población de estudio el porcentaje de casos con edades maternas sobre 30 años era similar al de casos menores de 30 años y sólo un $9 \%$ presentaba edades mayores de 40 años.

Respecto al manejo de estas patologías implica un análisis costo-eficiencia importante pues un $50 \%$ de los casos requirió manejo en unidad de paciente crítico y un $15 \%$ de los casos requiero manejo quirúrgico, mientras que un $30 \%$ de los casos egresó muerto y menos del $45 \%$ egresó vivo a su domicilio. Es necesario realizar un estudio acabado de las condicionantes asociadas, la necesidad de manejo multidisciplinario y en definitiva el manejo mas adecuado para este grupo de pacientes.

Podemos concluir que en base a un promedio de 11 casos anuales de malformaciones craneofaciales y siendo el HHHA un centro GES para pacientes con fisuras labiopalatinas, es necesario mantener un equipo de trabajo activo en el manejo de la patología. Es necesario determinar las mejores condiciones de manejo de los pacientes a fin de optimizar los recursos existentes. Finalmente, podemos señalar que las fisuras labiopalatinas son las patologías mas prevalentes dentro de las malformaciones craneofaciales.

MITTERSTEINER, D. \& OLATE, S. Craniofacial malformation in a high complexity regional hospital. Int. J. Odontostomat., 7(2):179-184, 2013.

ABSTRACT: Craniofacial malformations are complex pathologies that affect life in different ways. The objective of this research is to identify the prevalence of craniofacial malformations and to determine their management at the Hospital Hernán Henriquez Aravena (HHHA) in Temuco. This research was realized in the neonatology unit of this hospital, studying all admissions with some craniofacial malformation diagnostic during the period between January 2006 and August 2010. We studied variables from patient, mother, diagnostic and treatment. The information was analyzed in a descriptive way. We found 44 cases with craniofacial malformations, with $56.82 \%$ male and $22.73 \%$ belonging to the Mapuche ethnic group. The majority of the cases had weight according to their gestational age. More than $30 \%$ of the cases were preterm. Further, $52.27 \%$ were under 30 years old. $50 \%$ were managed in the critically ill patient unit, $16 \%$ of the cases needed surgery during their hospital staying in the neonatology unit and nearly $70 \%$ of the cases were discharged alive to their home, other unit or hospital. We concluded that cleft lip and palate was the most prevalent craniofacial malformation and multidisciplinary management for complex cases is necessary. 11 cases in one year requires an active team in the area.

KEY WORDS: malformation, cleft palate, craniofacial syndrome. 


\section{REFERENCIAS BIBLIOGRÁFICAS}

Alderman, B. W.; Lammer, E. J.; Joshua, S. C.; Cordero, J. F.; Ouimette, D. R.; Wilson, M. J. \& Ferguson, S. W. An epidemiologic study of craniosynostosis: risk indicators for the occurrence of craniosynostosis in Colorado. Am. J. Epidemiol., 128(2):431-8, 1988.

Felemovicius, J. \& Ortiz-Monasterio, F. Management of the impaired adult cleft patient: The last chance. Cleft Palate Craniofac. J., 41(5):550-8, 2004.

González, T.; Arias, J.; Lassaletta, L. \& Martorell, V. Malformaciones Craneofaciales y su Tratamiento. Nascer e Crescer, 12(4):S293-6, 2003.

INE-MIDEPLAN. Estadísticas sociales de los pueblos indígenas en Chile, Censo 2002. Santiago de Chile, MIDEPLAN, 2005.

MINSAL. Guía Clínica fisura labiopalatina. Santiago, Chile, MINSAL, 2009.

Múfalo, P.; Kaizer, Rde O.; Dalben, Gda S. \& de Almeida, A. L. Comparison of Periodontal Parameters in Individuals with Syndromic Craniosynostosis. J. Appl. Oral Sci., 17(1):13-20, 2009.

Navas, M. Descripción y prevalencia de malformaciones craneales y craneofaciales en el hospital nacional de niños Dr. Carlos Sáenz Herrera, Caja Costarricense de Seguro Social, durante el período 2002-2004. Rev. Cient. Odontol., 4(1):24-9, 2008.

Nazer, J.; Villa, J. J.; Van Deer Baars, R. \& Cifuentes, L. Incidencia de Labio Leporino y Paladar Hendido en Latinoamérica. Período 1982-1990. Pediatría (Santiago de Chile), 37(1-2):13-9, 1995.

Nazer, J.; Ramirez, M. C. \& Cifuentes, L. 38 Años de vigilancia epidemiológica de labio leporino y paladar hendido en la maternidad del Hospital Clínico de la Universidad de Chile. Rev. Méd. Chile, 138(5):567-72, 2010.

Nazer, J.; Hubner, M.; Catalán, J. \& Cifuentes, L. Incidencia de labio leporino y paladar hendido en la Maternidad del Hospital Clínico de la Universidad de Chile y en las maternidades chilenas participantes en el Estudio Colaborativo Latino Americano de Malformaciones Congénitas (ECLAMC) período 1991-1999. Rev. Méd. Chile, 129(3):285-93, 2001.

Nazer, J.; Pardo, R. \& Cifuentes, L. Prevalencia al nacimiento de malformaciones congénitas y de menor peso de nacimiento en hijos de madres adolescentes. Rev. Méd. Chile, 131(10):1165-72, 2003.
Olavarría, M. Acceso a la salud en Chile. Acta Bioeth., 11(1):47-64, 2005.

Roberts, R. M. \& Shute, R. Children's experience of living with a craniofacial condition: Perspectives of children and parents. Clin. Child Psychol. Psychiatry, 16(3):317-34, 2010.

Sepúlveda, G.; Palomino, H. \& Araya J. Prevalencia de fisura labiopalatina e indicadores de riesgo: Estudio de la población atendida en el Hospital Clínico Félix Bulnes de Santiago de Chile. Rev. Esp. Cirug. Oral Maxilofac., 30(1):17-25, 2008.

Sze, R. W.; Paladin, A.; Lee, S. \& Cunningham, M. Hemifacial microsomia in pediatric patients. Asymetric abnormal development of the first and second branquial arches. Am. Roentgen J., 178(6):1523-30, 2002.

Dirección para Correspondencia

Prof. Dr. Sergio Olate

Unidad de Cirugía Oral y Maxilofacial

Facultad de Odontología

Universidad de La Frontera

Claro Solar No 115, Temuco

CHILE

Telefono: $56-45-325000$

Email: sergio.olate@ufrontera.cl

Recibido : 15-08-2012

Aceptado: 22-03-2013 